\title{
The Bs20x22 anti-CD20-CD22 bispecific antibody has more lymphomacidal activity than do the parent antibodies alone
}

\author{
Joseph M. Tuscano $\cdot$ Yunpeng Ma • \\ Shiloh M. Martin · Jason Kato $\cdot$ Robert T. O'Donnell
}

Received: 21 September 2010/ Accepted: 18 January 2011/Published online: 24 February 2011

(C) The Author(s) 2011. This article is published with open access at Springerlink.com

\begin{abstract}
Previous studies have shown that bispecific antibodies that target both CD20 and CD22 have in vivo lymphomacidal properties. We developed a CD20-CD22 bispecific antibody (Bs20x22) from anti-CD20 and the anti-CD22 monoclonal antibodies (mAb), rituximab and HB22.7, respectively. Bs20x22 was constructed using standard methods and was shown to specifically bind $\mathrm{CD} 20$ and CD22. In vitro cytotoxicity assays showed that Bs20x22 was three times more effective than either parent $\mathrm{mAb}$ alone and twice as effective as a combination of both parent $\mathrm{mAb}$ used at equimolar concentrations. Bs20x22 was also nearly four times more effective at inducing apoptosis than either $\mathrm{mAb}$ alone. Examination of the MAPK and SAPK signaling cascades revealed that Bs20x22 induced significantly more p38 phosphorylation than either $\mathrm{mAb}$ alone. In an in vivo human NHL xenograft model, treatment with Bs20x22 resulted in significantly greater tumor shrinkage and improved overall survival when compared to either $\mathrm{mAb}$ alone or treatment with a combination of HB22.7 and rituximab. The effect of the initial tumor volume was assessed by comparing the efficacy of Bs20x22 administered before xenografts grew versus treatment of established tumors; significantly,
\end{abstract}

J. M. Tuscano (ه) · Y. Ma · S. M. Martin · J. Kato ·

R. T. O'Donnell

Division of Hematology and Oncology,

Department of Internal Medicine, University of California,

Davis, Cancer Center, 4501 X Street, Suite 3016,

Sacramento, CA 95817, USA

e-mail: joseph.tuscano@ucdmc.ucdavis.edu

J. M. Tuscano - R. T. O’Donnell

Veteran's Administration Northern California Healthcare

System, Los Angeles, CA, USA greater efficacy was found when treatment was initiated before tumors could become established.

Keywords CD22 - Lymphoma - Monoclonal antibody · Diabody $\cdot$ Bispecific

\section{Introduction}

Approximately 250,000 people in the United States have non-Hodgkin's lymphoma (NHL), an $80 \%$ increase since 1970; NHL is the sixth most common cause of cancerrelated deaths in the United States [1]. NHL is a heterogeneous group of malignancies, the majority of which are of B-lymphocyte origin (B-NHL). While standard cytotoxic chemotherapy is initially effective, resistance often develops and the dose is often limited by toxicity $[2,3]$.

MAb-based therapy has enormous promise. The chimeric anti-CD20 mAb, rituximab, produces overall and complete remission rates of 50 and $10 \%$, respectively, in patients with relapsed follicular NHL [4]. Rituximab is also used in combination with several cytotoxic chemotherapy regimens for both indolent and aggressive NHL and its addition has led to improvements in overall survival [5]. Despite these advances, the majority of patients with NHL eventually succumb to their disease. Because of the safety and efficacy of mAbs like rituximab, new mAbs are being tested to see if they are effective, synergistic with rituximab, or useful against drug-resistant NHL.

CD22 is a B-lymphocyte-specific glycoprotein adhesion molecule that can bind multiple types of hematopoietic cells and transduces signals to the cell's interior, resulting in a cascade of phosphorylation events [6-8]. Nearly all mature B-cells express CD22, although it disappears upon differentiation into plasma cells. Most B-NHL express CD22 
[9-11], making CD22 a promising therapeutic target. The extracytoplasmic portion of CD22 contains seven Ig-like domains [7]. The two amino-terminal Ig domains mediate cell adhesion to sialic-acid bearing ligands [12-14]. Anti-CD22 mAbs, such as HB22.7, that bind the two aminoterminal Ig domains of CD22 and specifically block the interaction of $\mathrm{CD} 22$ with its ligand induce proliferative responses in primary B-cells, but apoptotic responses in neoplastic B-cells $[8,15]$. In contrast, anti-CD22 mAbs that do not block ligand binding have only modest functional effects [15]. We previously characterized several anti-CD22 $\mathrm{mAbs}$ that have unique signaling properties, pro-apoptotic effects, and significant in vivo lymphomacidal capacity [16].

The combination of epratuzumab (anti-CD22) and rituximab (anti-CD20) is efficacious [17-20]; however, this combination must be administered sequentially, greatly increasing the infusion time needed to treat a patient. This makes a bispecific antibody (BsAb) that simultaneously targets both CD20 and CD22 an attractive alternative to the use of two different mAb. BsAbs can be designed to crosslink two antigens on the same cell type, such as CD20 and CD22 on B-NHL. CD20/CD22 BsAbs have been previously characterized, tested, and found to be promising against NHL $[25,26]$. These BsAbs use veltuzumab (antiCD20) and epratuzumab (anti-CD22) as a platform. In contrast, we constructed a BsAb (Bs20x22) using rituximab (anti-CD20) and HB22.7 (anti-CD22). The choice of which anti-CD22 mAb to use is critical. We previously demonstrated that anti-CD22 mAbs that block CD22 ligand binding have greater efficacy than those that do not block ligand binding [27]. Epratuzumab is rapidly internalized into NHL cells and causes CD22 phosphorylation, but does not block CD22 ligand binding, does not initiate CD22mediated signal transduction or apoptosis, and does not demonstrate any direct cytotoxic or cytostatic effects [28]. In contrast, HB22.7 does block CD22 ligand binding, initiates CD22-mediated signal transduction, demonstrates direct cytotoxic effects and has been found to improve survival and decrease tumor volume in a human NHL xenograft mouse model $[16,27,29]$.

Herein, we describe the construction of a CD20/CD22targeted BsAb (Bs20x22) using a rituximab/HB22.7 platform, its cell-binding characteristics, induced signaling patterns, and pre-clinical efficacy in a human NHL xenograft model.

\section{Materials and methods}

\section{Materials}

The following materials were used: Sephadex G-25, Superdex 200, iodoacetamide, o-phenylenedimaleimide (PDM), 2-mercaptoethanol, sodium acetate, sodium orthovanadate, 2-glycerophosphate, bovine serum albumin (BSA), Tween20, CelLytic M lysis buffer, dimethylformamide (DMF), Coomassie Brilliant Blue R, protease inhibitor cocktail tablets and ethylenediaminetetraacetic acid (EDTA) (Sigma Chemical Co.,St. Louis, MO), goat anti-mouse immunoglobulins fluorescein conjugate (goat anti-mouse Ig-FITC) (Biosource, Camarillo, CA), mouse anti-human $\operatorname{IgG}$ $(\mathrm{H}+\mathrm{L})$ Texas Red conjugate (Rockland Immunochemicals; Gilbertsville, PA), anti-phospho-JNK, anti-JNK, antiphospho-ERK1/2, anti-ERK1/2, anti-phospho-p38, and anti-p38 antibodies (Cell Signaling Technology, Danvers, MA), anti-mouse HRP antibody (Dako North America, Inc, Carpentaria, CA), polycaspases FLICA (FAM-VAD-FMK) kit (Immunochemistry Technologies, LLC, Bloomington, $\mathrm{MN}), \mathrm{BCA}^{\mathrm{TM}}$ protein assay kit, Silver SNAP II Stain Kit, and ImmunoPure $\mathrm{F}\left(\mathrm{ab}^{\prime}\right)_{2}$ Preparation Kit (Pierce, Rockford, IL), RPMI 1640 medium, DMEM medium, penicillin-streptomycin and fetal bovine serum (FBS) (Life Technologies, Carlsbad, CA), Rituximab (Rituxan) (Genentech (South San Francisco, CA). The anti-CD22 mAb, HB22.7, was purified from ascites and has been previously characterized [8]. All chemicals were of analytical grade purity.

\section{Cell lines}

The CD20/CD22 double positive human Burkitt's B-cell lymphoma lines, Raji (ATCC CCL-86) and Ramos (ATCC CRL-1596), and the CD20/CD22 double negative human embryonic kidney cell line 293T (ATCC CRL-11268) were purchased from American Type Culture Collection (Rockville, MD). All cells were thawed and grown in RPMI-1640 (Raji, Ramos) or DMEM (293T) supplemented with $10 \%$ FBS, 50 units $/ \mathrm{ml}$ penicillin $\mathrm{G}$, and $50 \mu \mathrm{g} / \mathrm{ml}$ streptomycin sulfate. Cells were maintained in tissue culture flasks at $37^{\circ} \mathrm{C}$ in $5 \% \mathrm{CO}_{2}$ and $90 \%$ humidity. After two passages, multiple vials were re-frozen and stored in liquid nitrogen for future use. Fresh vials of cells are periodically thawed and used for in vitro experiments to ensure that changes to cells have not occurred over time/passages in culture. For xenograft studies, a fresh vial of Raji cells was thawed 7-10 days before tumor cell implantation.

\section{Bs20x22 construction}

$\mathrm{F}(\mathrm{ab})_{2}$ fragments of rituximab and HB22.7 were made using ImmunoPure $\mathrm{F}\left(\mathrm{ab}^{\prime}\right)_{2}$ Preparation Kit (Pierce) according to manufacturer's instructions. For quality control, protein containing fractions were run on a $10 \%$ SDSPAGE gel under non-reducing conditions and silver stained using Silver SNAP II Stain Kit (Pierce). F(ab') ${ }_{2}$ containing fractions were pooled and concentrated using a 10,000 MWCO centrifugal filter (Millipore). Each $\left.\mathrm{F}(\mathrm{ab})_{2}\right)_{2}$ was reduced to $F(a b)$-thiol by adding 2-mercaptoethanol to a 
final concentration of $20 \mathrm{mM}$ at $30^{\circ} \mathrm{C}$ for $30 \mathrm{~min}$. Samples were chilled on ice and then passed over chilled Sephadex G25 columns equilibrated in $50 \mathrm{mM}$ sodium acetate/ $0.5 \mathrm{mM}$ EDTA $\mathrm{pH}$ 5.3. Protein-containing fractions were pooled. The HB22.7 Fab-thiol was then maleimidated for $30 \mathrm{~min}$ in a methanol ice bath by addition of $1 / 2$ volume of pre-chilled $12 \mathrm{mM}$ o-PDM (dissolved in DMF). HB22.7maleimidated-Fab was then passed over a chilled Sephadex G25 column equilibrated in $50 \mathrm{mM}$ sodium acetate/ $0.5 \mathrm{mM}$ EDTA $\mathrm{pH}$ 5.3. Protein-containing fractions were pooled and immediately added to rituximab-thiol-Fab in a 1:1 molar ratio. The reaction was placed under a constant stream of nitrogen gas and stirred gently for $18 \mathrm{~h}$ at $4^{\circ} \mathrm{C}$. The $\mathrm{pH}$ was adjusted to $\mathrm{pH} 8.0$ using $1 \mathrm{M}$ Tris- $\mathrm{HCl} \mathrm{pH}$ 8.0 , followed by the addition of 2-mercaptoethanol to a final concentration of $20 \mathrm{mM}$. The reaction was incubated for $30 \mathrm{~min}$ at $30^{\circ} \mathrm{C}$ and then alkylated by adding iodoacetamide to a final concentration of $25 \mathrm{mM}$. The mixture was then passed over a Superdex 200 column equilibrated in PBS pH 7.4, and fractions were collected. Individual fractions were run on $10 \%$ SDS-PAGE gels under nonreducing conditions and stained with Coomassie Brilliant Blue (Sigma) or Silver Snap II Kit (Pierce). F(ab') $)_{2}$ containing fractions (Bs20x22) were identified and pooled.

\section{Cell-binding studies}

The first set of binding studies was performed on 293T cells (CD20/CD22 negative) transfected with CD20 or CD22. Transfection was performed using a standard calcium phosphate-mediated transfection protocol. Cells were used for binding studies $24-48 \mathrm{~h}$ after transfection. CD20-transfected or CD22-transfected cells $\left(0.5 \times 10^{6}\right.$ per sample $)$ were trypsinized, washed twice in FACS buffer (PBS supplemented with $0.2 \%$ FBS), and stained with HB22.7, rituximab, or Bs20x22 (5 $\mathrm{g}$ in $100 \mu \mathrm{L}$ FACS buffer). As controls, untransfected cells were also stained with HB22.7, rituximab, or Bs20x22 in the same manner. Cells were incubated for $20 \mathrm{~min}$ on ice and then washed 4 times with FACS buffer. Cells were stained with secondary antibodies (1 $\mu \mathrm{g}$ in $100 \mu \mathrm{L}$ FACS buffer) as follows: HB22.7-treated samples were stained with goat anti-mouse Ig-FITC, rituximab-treated samples were stained with goat anti-human IgG-FITC, and Bs20x22-treated samples were stained with either goat anti-mouse Ig-FITC (to detect HB22.7 component) or goat anti-human IgG-FITC (to detect rituximab component). As additional controls, CD20-transfected, CD22-transfected, and untransfected cells were treated with secondary antibodies only (no HB22.7, rituximab, or Bs20x22 staining). Cells were incubated on ice for $15 \mathrm{~min}$ and then washed 4 times in FACS buffer. Images were acquired by fluorescence microscopy (Olympus Motorized Reflected Fluorescence System BX61).
The second set of binding studies was performed using CD20/CD22 double positive Raji and Ramos cells. Cells $\left(0.5 \times 10^{6}\right.$ per sample $)$ were washed and stained with HB22.7, rituximab, and $\mathrm{Bs} 20 \times 22$ as described earlier. Secondary antibodies used in this experiment were as follows: HB22.7-treated samples were stained with goat antimouse Ig-FITC, rituximab-treated samples were stained with goat anti-human IgG-TxRd, and Bs20x22-treated samples were treated with either goat anti-mouse Ig-FITC (to detect HB22.7 component) or goat anti-human IgGTxRd (to detect rituximab component). As additional controls, cells were treated with secondary antibodies only (no HB22.7, rituximab, or Bs20x22). Images were acquired by fluorescence microscopy.

\section{In vitro cytotoxicity assay}

Raji or Ramos cells $\left(2-2.5 \times 10^{4}\right.$ per sample) were plated on triplicate in 96-well round bottom plates in a volume of $100 \mu \mathrm{L}$ per well. Cells were treated for $1 \mathrm{~h}$ with HB22.7, rituximab, $\mathrm{HB} 22.7$ plus rituximab, or $\mathrm{Bs} 20 \times 22$ to final concentrations of $0,0.005,0.05,0.5,5,50,100$, or 200 $\mu \mathrm{g} / \mathrm{mL}$. Control cells received media only. After treatment, plates were washed 3 times in media and then incubated at $37^{\circ} \mathrm{C}$ in $5 \% \mathrm{CO}_{2}$ and $90 \%$ humidity for 5 days. Viability was assessed by trypan blue exclusion; results were expressed as $\%$ of control (untreated cells).

In vitro apoptosis assay

Raji or Ramos cells $\left(1 \times 10^{6}\right)$ were plated in a volume of $1 \mathrm{~mL}$ per well. Cells were treated with $\mathrm{HB} 22.7(40 \mu \mathrm{g} / \mathrm{mL})$, rituximab $(40 \mu \mathrm{g} / \mathrm{mL}), \mathrm{HB} 22.7$ plus rituximab $(40 \mu \mathrm{g} / \mathrm{mL}$ per $\mathrm{mAb}$ for a total of $80 \mu \mathrm{g} / \mathrm{mL})$, or Bs $20 \times 22(80 \mu \mathrm{g} / \mathrm{mL})$. Control cells received media only. All samples were plated in triplicate. Cells were incubated at $37^{\circ} \mathrm{C}$ in $5 \% \mathrm{CO}_{2}$ and $90 \%$ humidity for $4 \mathrm{~h}$. Cells were then washed and stained with the Polycaspases FLICA (FAM-VAD-FMK) kit (Immunochemistry Technologies, LLC) according to manufacturer's instructions. Cells were also stained with Hoechst stain and propidium iodide (included in the kit) according to instructions. Cells were then analyzed by fluorescence microscopy (Olympus Motorized Reflected Fluorescence System BX61). Cells exhibiting positive propidium iodide staining (dead cells) were excluded from further analysis. In each $20 \times$ field, the total number of cells (positive Hoechst stain) and the number of apoptotic cells (positive FAM-VAD-FMK stain) were counted. Three fields were counted for each sample. The percentage of apoptotic cells was calculated by dividing the number of apoptotic cells in a given field by the total number of cells in that field. Results were graphed as percentage of control (\% apoptotic cells treated/\% apoptotic cells untreated). 


\section{Western blotting}

Ramos cells $\left(2 \times 10^{7}\right.$ per treatment $)$ were treated with one of the following: rituximab (100 $\mu \mathrm{g} / \mathrm{mL}), \mathrm{HB} 22.7$ $(100 \mu \mathrm{g} / \mathrm{mL})$, or Bs20x22 $(100 \mu \mathrm{g} / \mathrm{mL})$ in growth media. Two different preparations of Bs20x22 (prepared approximately 4 months apart) were tested. As a positive control, one group of cells was treated with anti-IgM $(50 \mu \mathrm{g} / \mathrm{mL})$. Untreated cells were used as a negative control. Cells were treated for $15 \mathrm{~min}$ at $37^{\circ} \mathrm{C}$. After treatment, cells were centrifuged $(400 \mathrm{~g})$ and cell pellets resuspended in $125 \mu \mathrm{L}$ of Cell Lytic M lysis buffer supplemented with a protease inhibitor cocktail tablet, sodium orthovanadate, and 2-glycerophosphate. Cells were lysed on ice for $30 \mathrm{~min}$ with occasionally vortexing. Lysates $(50 \mu \mathrm{g}$ protein per lane) were run on a $10 \%$ SDS-PAGE gel, followed by transfer to a nitrocellulose membrane. Membranes were blocked for $1 \mathrm{~h}$ at room temperature in 5\% BSA in TBS-T, rinsed in TBS-T, and then incubated at $4{ }^{\circ} \mathrm{C}$ overnight in primary antibodies (anti-phospho-JNK, anti-phosphoERK1/2, or anti-phospho p38) diluted 1:1,000 in 5\% BSA in TBS-T. Membranes were washed 3 times in TBS-T and then incubated for $1 \mathrm{~h}$ at room temperature with antimouse HRP conjugate diluted $1: 10,000$ in 5\% BSA in TBS-T. Membranes were washed 4 times in TBS-T and then probed with Advanced ECL detection reagent. To determine total JNK, ERK1/2, and p38 levels, after detection of phospho proteins, each membrane was stripped and then re-probed with either anti-JNK, anti-ERK1/2, or anti-p38 antibodies (1:1,000 dilution).

\section{Xenograft studies}

Six- to eight-week-old female Balb/c nude mice were obtained from Harlan Sprague-Dawley (Indianapolis, IN) and maintained in micro-isolation cages under pathogenfree conditions in the UC Davis animal facility. Three days after whole body irradiation (400 rads), $0.5 \times 10^{6}$ Raji cells were implanted subcutaneously on the left flank. One day after tumor implantation, mice were randomly divided into 5 groups ( $n=6-8$ per group): rituximab only, HB22.7 only, combination rituximab/HB22.7, Bs20x22, or PBS (control). Mice were administered treatment on day 14, 21, and 28 after tumor implantation. Each dose was $140 \mu \mathrm{g}$ $(0.047 \mu \mathrm{mol})$ total $\mathrm{mAb}$ (thus, the combination group received $70 \mu \mathrm{g}$ of rituximab and $70 \mu \mathrm{g}$ of $\mathrm{HB} 22.7$, for a total of $140 \mu \mathrm{g})$. PBS was administered at a volume equivalent to the volume received by the Bs20x22 group. An additional group of mice $(n=8)$ were administered Bs20x22 treatment on day 1, 7, and 14 after tumor implantation (before tumors had time to become established). All treatments were administered via the tail vein. All xenograft studies were repeated using the same sample size for validation and statistical purposes. Tumors were measured twice per week using a caliper, and tumor volumes were calculated using the equation: (length $\times$ width $\times$ depth $\times 0.52$. Mice were euthanized when the tumor reached $15 \mathrm{~mm}$ in any dimension or $1,500 \mathrm{~mm}^{3}$. For statistical purposes, the volume prior to euthanasia was carried forward until completion of the study.

\section{Statistical analysis}

In vitro cytotoxicity data and apoptosis data were analyzed by a two-tailed, unpaired Student's t-test. Tumor volume data were analyzed using Kaplan-Meier curves. For this analysis, an "event" was defined as tumor volume reaching $400 \mathrm{~mm}^{3}$ or greater. Each individual mouse was ranked as a 1 (event occurred) or a 0 (event did not occur) and the time to event (in days) was determined. When an individual was ranked as 0 (event did not occur), a time to event of 88 days (number of days in the 12.5 week study) was recorded. Chi-squared and $P$ values were determined by the Log-rank test. All statistical analysis was performed using GraphPad Prism software (San Diego, CA). A $P$ value of $<0.05$ was considered significant.

\section{Results}

Bs20x22 exhibits cell binding similar to its parent mAbs

To determine if the CD20/CD22 bispecific antibody (Bs20x22) maintained binding characteristics similar to the parent mAbs, 293T cells were transfected with either CD20 or CD22 and stained with Bs20x22 or a parent mAb. Bs20x22 exhibited the same staining as rituximab on CD20-transfected cells (Fig. 1a, b) and the same staining as HB22.7 on CD22-transfected cells (Fig. 1c, d). Untransfected 293T cells showed no rituximab, HB22.7, or Bs20x22 staining (data not shown). On CD20/CD22-positive Ramos cells, Bs20x22 also exhibited the same staining as each parent $\mathrm{mAb}$ (Fig. 1 e, f). Staining results were verified in the CD20/CD22-positive cell line, Raji.

Bs20x22 exhibits greater in vitro cytotoxicity than the parent mAbs

The effect of Bs20x22 on in vitro cell viability was then assessed. Raji cells were treated with each parent $\mathrm{mAb}$ alone, combination rituximab plus HB22.7, or Bs20x22 for $1 \mathrm{~h}$, and then washed; cell viability was assessed 5 days later. As shown in Fig. 2a, both Bs20x22 and the rituximab plus HB22.7 combination demonstrated greater cytotoxicity than either parent $\mathrm{mAb}$ alone. In addition, 
Fig. 1 Transfected 293T or Ramos cells were stained with either rituximab, HB22.7, or Bs20x22, then washed and stained with the appropriate anti-human or anti-mouse fluorescent mAb. a CD20transfected 293T cells + rituximab. b CD20-transfected $293 \mathrm{~T}$ cells + Bs20x22.

c CD22-transfected 293T cells + HB22.7. d CD22transfected 293T cells + Bs20x22. e Ramos + Bs20x22 + anti-human Ig-TexasRed. f Ramos + Bs20x22 + anti-mouse Ig-FITC
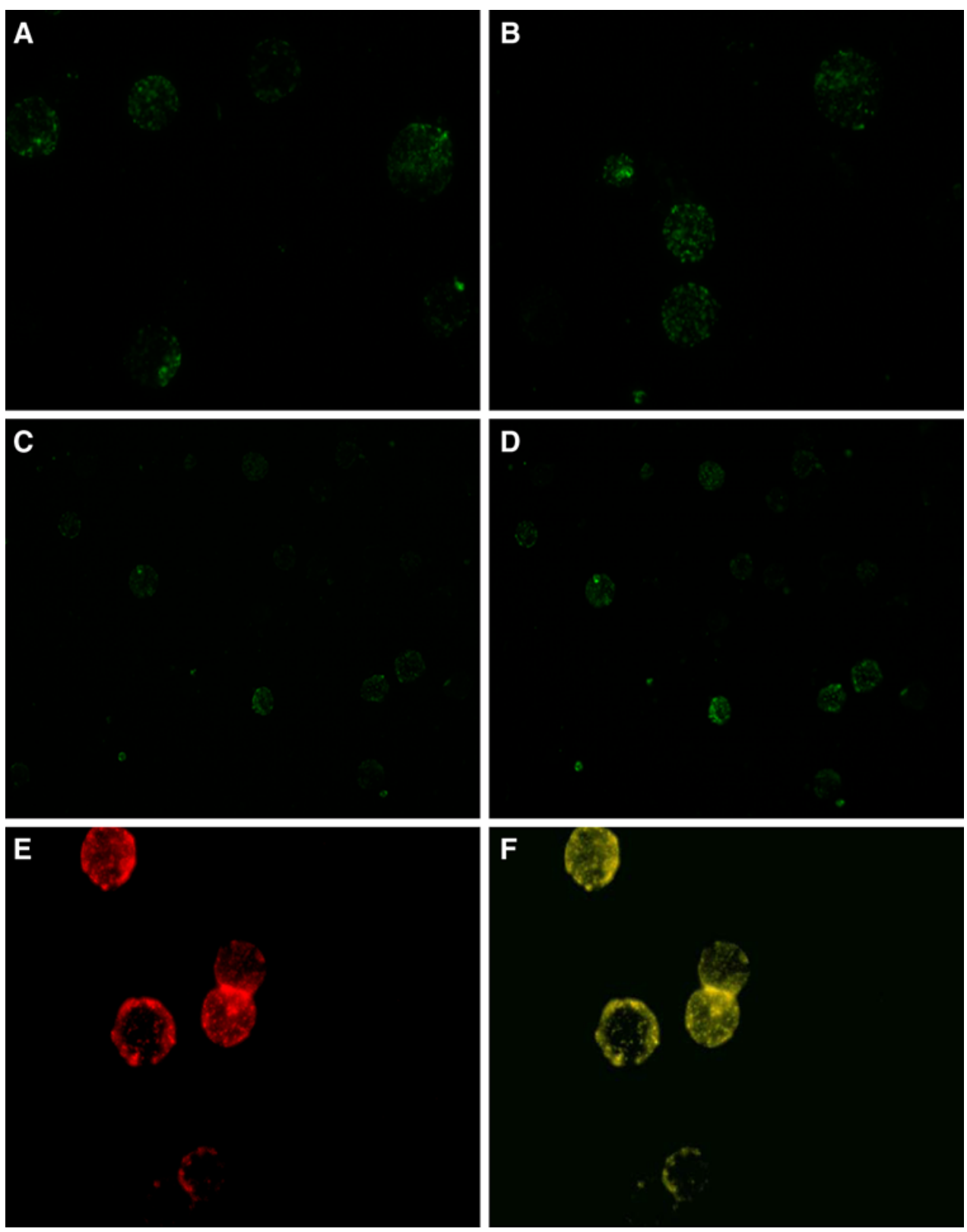

Bs20x22 was more cytotoxic than higher doses of the rituximab/HB22.7 combination (Fig. 2a). At the 50, 100, and $200 \mu \mathrm{g} / \mathrm{mL}$ doses, Bs20x22 treatment resulted in 28 , 16 , and $11 \%$ cell viability, respectively, compared with combination rituximab/HB22.7-treated cell viabilities of 35, 31, and 27\%. Similar results were also seen using Ramos cells (data not shown). To determine if the increase in cytotoxicity was due to apoptosis, Raji and Ramos cells were treated as described earlier and apoptosis was assessed using a polycaspase FLICA kit. The percent of Raji and Ramos cells that underwent apoptosis was greatest for Bs20x22 treatment (78 and 74\%) compared with combination rituximab plus HB22.7 (45 and
43\%), rituximab (35 and 34\%), and HB22.7 (24 and 22\%) (Fig. 2b).

Bs20x22 activates p-38 to a greater degree than either parent $\mathrm{mAb}$

To determine if Bs20x22 stimulated similar signaling pathways as the parent mAbs, Ramos cells were treated with rituximab, $\mathrm{HB} 22.7$, or $\mathrm{Bs} 20 \times 22$ for $15 \mathrm{~min}$, then washed and lysed. The lysates were analyzed by Western blot for phosphorylation/activation of MAP kinases p-38, JNK, and ERK1/2. Cross-linking the B-cell receptor (BCR) with anti-IgM was the positive control. Treatment with 

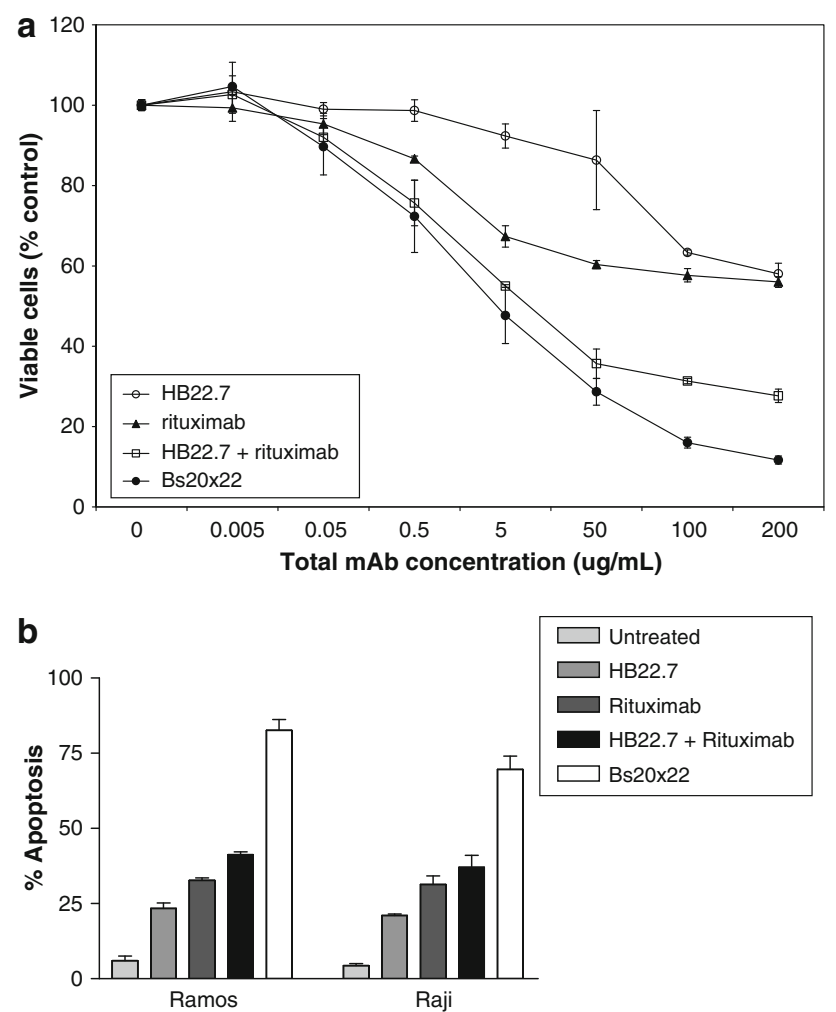

Fig. 2 Raji cells were treated with rituximab (filled triangle), HB22.7 (empty circle), combination rituximab/HB22.7 (empty square), or Bs20x22 (filled circle) and assessed for cell viability (a). Raji and Ramos cells were assessed for apoptotic induction (b). Error bars represent the standard deviation $* P$ value $<0.02$ (Bs20x22 vs rituximab/HB22.7). $* * P$ value $<0.01 \quad$ (Bs20x22 vs rituximab/ HB22.7). ***P value $<0.01$ (Bs20x22 vs all groups)

Bs20x22 resulted in significant activation of $\mathrm{p} 38$, while treatment with either parent mAb did not (Fig. 3). Neither JNK nor ERK1/2 were activated by Bs20x22, rituximab, or HB22.7.

\section{Bs20x22 improves survival more than either parent $\mathrm{mAb}$}

Nude mice bearing Raji xenografts were treated with rituximab, HB22.7, combination rituximab plus HB22.7, or Bs20x22 on days 14, 21, and 28 after tumor implantation. As shown in Fig. 4a, treatment with Bs20x22 resulted in the smallest tumor volume of any group, although statistical significance was achieved only for the Bs20x22 versus control and the Bs20x22 versus HB22.7 comparisons (Table 1). Mice treated with the combination of rituximab and HB22.7 also had smaller tumor volumes than mice treated with either parent $\mathrm{mAb}$ alone (Fig. $4 \mathrm{a}$ ). By the end of the study, Bs20x22-treated mice had tumors that were, on average, $50 \%$ smaller than tumors from combination rituximab/HB22.7-treated mice (Fig. 4a). As shown in Fig. 4b, Bs20x22-treated mice exhibited the greatest

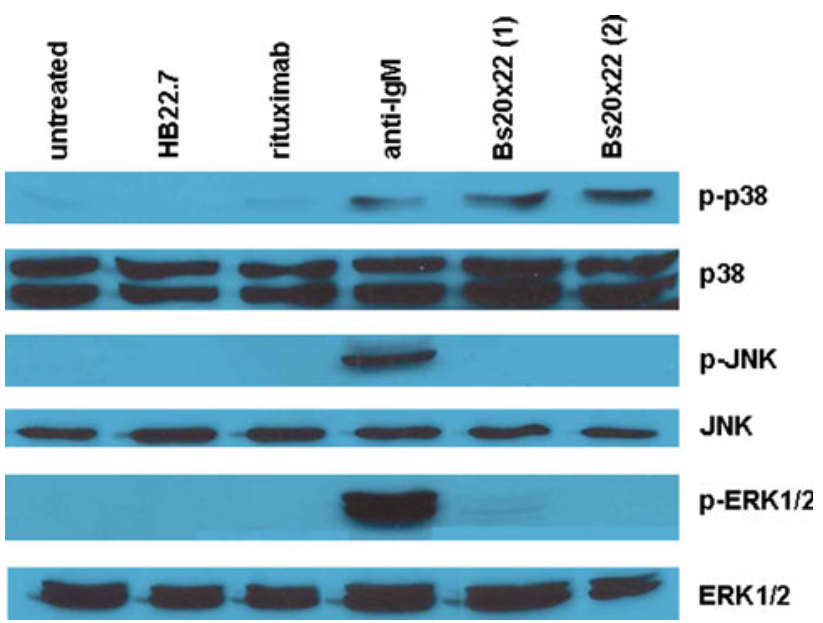

Fig. 3 Ramos cells were treated with rituximab, HB22.7, or two different preparations (labeled 1 and 2) of Bs20x22. MAP kinase activation (p-38, JNK, ERK1/2) was analyzed by Western blot. AntiIgM was included as a positive control. This represents a consistent and representative experiment, which was done in duplicate

survival rate at the end of the study (88\%) compared with combination rituximab plus HB22.7 (75\%), rituximab (50\%), HB22.7 (25\%), and control (0\%).

Previous studies with both HB22.7 and rituximab had demonstrated greater efficacy when treating smaller tumor volumes [16, 27, 29]; therefore, we compared pre-emptive (prior to the development of visible tumors) Bs20x22 treatment (days 1,7 , and 14 post-implantation) to Bs20x22 treatment of established tumors (days 14, 21, and 28 postimplantation). By the end of the study, mice treated preemptively with Bs20x22 had tumors that were $89 \%$ smaller than those in the established tumor-Bs20x22 treatment group (Fig. 4c).

\section{Discussion}

Other groups have tested BsAbs that target CD20 and CD22 using veltuzumab and epratuzumab, respectively, and these BsAbs demonstrated enhanced efficacy when compared to the parent mAbs $[25,26]$. While the veltuzumab/epratuzumab bispecific mAb had enhanced in vitro cytotoxicity, this was dependent upon hypercrosslinking with a secondary antibody and this did not translate into better survival when compared to the parent $\mathrm{mAb}$ in in vivo. To improve cross-linking, a hexavalent veltuzumab/epratuzumab-derived CD20/CD22 bispecific mAb was developed and tested in the same in vitro and in vivo models. The hexavalent antibody demonstrated enhanced apoptotic induction, and this did translate into improved survival (approximately 35-40\%). However, the improved survival depended upon host immune effector mechanisms. This was demonstrated by the fact that the survival 

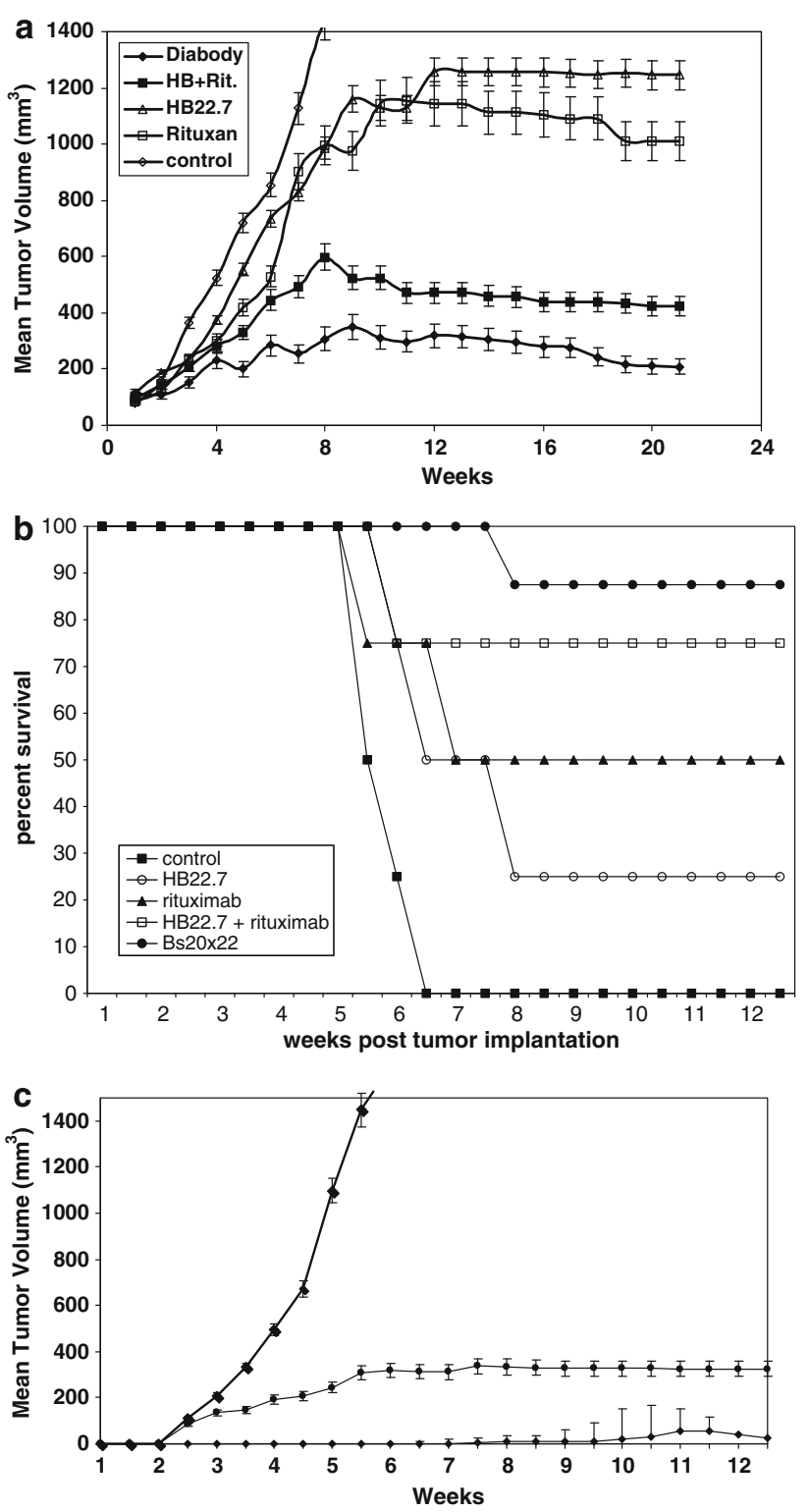

Fig. 4 Mice bearing Raji NHL xenografts were treated with rituximab (empty square), HB22.7 (empty triangle), combination rituximab/HB22.7 (solid square), Bs20x22 (filled diamond), or PBS control (empty diamond). Mice were assessed twice weekly for tumor volume (a) and survival (b). Mice bearing Raji NHL xenografts were treated with Bs20x22 before ("pre-emptive", filled triangle) or after ("established", filled circle) tumors were established and compared with untreated controls (filled squares) (c). Replicate experiments were done with consistent results with the data presented representing an analysis of all mice studied. A total of 16 mice pre-treatment group were used with the error bars representing the standard deviation

advantage was abrogated by NK cell depletion, which is likely related to the fact that the hexavalent $\mathrm{mAb}$ contained an intact antibody and $\mathrm{Fc}$ component. However, the choice of anti-CD22 mAb is a critical one-different anti-CD22 mAbs exhibit distinct functional effects. The Tedder laboratory mapped and characterized CD22 ligand-binding
Table 1 Xenograft statistics: Tumor volume data (from Fig. 4a) were analyzed as described in "Materials and methods"

\begin{tabular}{lll}
\hline Comparison & Chi $^{2}$ & $P$ value \\
\hline Control vs. HB22.7 & 2.798 & 0.0944 \\
Control vs. rituximab & 2.561 & 0.1095 \\
Control vs. HB22.7 + rituximab & $\mathbf{4 . 3 1 0}$ & $\mathbf{0 . 0 3 7 9}$ \\
Control vs. Bs20x22 & $\mathbf{8 . 5 3 2}$ & $\mathbf{0 . 0 0 3 5}$ \\
HB22.7 vs. rituximab & 1.315 & 0.2515 \\
HB22.7 vs. HB22.7 + rituximab & 1.887 & 0.1696 \\
HB22.7 vs. Bs20x22 & $\mathbf{7 . 7 0 2}$ & $\mathbf{0 . 0 0 5 5}$ \\
Rituximab vs. HB22.7 + rituximab & 0.285 & 0.5933 \\
Rituximab vs. Bs20x22 & 1.636 & 0.2009 \\
Bs20x22 vs. HB22.7 + rituximab & 0.378 & 0.5385 \\
\hline
\end{tabular}

The $\mathrm{Chi}^{2}$ and $P$ value are shown for each comparison. Statistically significant comparisons $(P$ value $<0.05)$ are shown in bold

domains using a panel of anti-CD22 mAbs and found that several mAbs, such as HB22.7, completely blocked interaction with its ligand on all cell types examined, while other anti-CD22 mAbs, such as HB22.27, exhibited no blocking ability [12]. Subsequent studies used constructs expressing specific deletions of CD22 Ig-like domains (domains 1-4) to map where these mAbs bound within the extracytoplasmic portion of $\mathrm{CD} 22$, and the two aminoterminal domains ( 1 and 2 ) were found to be necessary for mAb-dependent ligand blocking [30]. The pro-apoptotic effects of these anti-CD22 mAbs were also studied, and there was a significant induction of apoptosis after crosslinking CD22 with the blocking mAb HB22.7 [8]. Little induction of apoptosis was seen with the non-blocking $\mathrm{mAb}$, HB22.27, demonstrating the distinct functional properties ligand blocking mAbs versus non-blocking mAbs [8]. Additionally, ligand blocking anti-CD22 mAbs are more effective than non-blocking anti-CD22 mAbs at initiating CD22-mediated signal transduction [31]. These studies demonstrate that ligand blocking anti-CD22 mAbs are unique and functionally distinguishable from other antiB-cell and even other anti-CD22 mAb.

In vitro studies demonstrated that epratuzumab was rapidly internalized into NHL cells and caused CD22 phosphorylation, but did not initiate CD22-mediated signal transduction or apoptosis and did not demonstrate any direct cytotoxic or cytostatic effects [28]. Epratuzumab binds to the Ig-like domain 3 of CD22 [32], making it unlikely than epratuzumab has ligand blocking properties. In contrast, the HB22.7 anti-CD22 mAb, binds domains 1 and 2 and does exhibit ligand blocking properties. In a pre-clinical NHL model, when HB22.7 was compared with another anti-CD22 mAb (HB22.27) that similar to epratuzumab, does not block CD22 ligand binding; HB22.7 had superior efficacy $[13,27]$. The superior efficacy of HB22.7 over the epratuzumab-like HB22.27 most likely 
occurred because the lymphomacidal properties of HB22.7 depend upon the targeting of the specific CD22 epitope (domains 1 and 2) bound by HB22.7. In addition, studies by the Tedder laboratory have demonstrated that the CD22 ligand-binding domain is required for in vivo B-cell survival [33], which could explain why anti-CD22 mAbs that bind to this ligand-binding domain have greater efficacy than anti-CD22 mAbs that bind to non-ligand-binding domains. For these reasons, we believe HB22.7 is a better platform than epratuzumab for CD22-based BsAbs. We constructed a CD20-CD22 BsAb (Bs20x22) using HB22.7 and rituximab as a platform and tested this BsAb in a preclinical NHL model.

To determine if Bs20x22 maintained similar binding characteristics as the parent mAbs, Ramos cells (CD20/ CD22 positive) and 293T cells transfected with either $\mathrm{CD} 20$ or CD22 were stained with Bs20x22, rituximab, or HB22.7. Ramos cells and CD20 transfected 293T cells exhibited Bs20x22 staining that was equivalent to rituximab (Fig. 1a, b, e). Similarly, Ramos cells and CD22 transfected 293T cells exhibited Bs20x22 staining that was equivalent to HB22.7 (Fig. 1c, d, f). Un-transfected 293T cells showed no rituximab, HB22.7, or Bs20x22 staining. These results indicate that Bs20x22 exhibits specific binding to both CD20 and CD22.

We next examined the in vitro cytotoxicity of Bs20x22. Both Bs20x22 and the rituximab/HB22.7 combination treatment demonstrated greater cytotoxicity than either parent $\mathrm{mAb}$ alone at doses of $0.5 \mu \mathrm{g} / \mathrm{mL}$ or higher (Fig. 2a). Bs20x22 was more cytotoxic than the rituximab/ HB22.7 combination treatment at lower doses. However, at higher doses (100-200 $\mu \mathrm{g} / \mathrm{mL})$, Bs20x22 was significantly more cytotoxic than the rituximab/HB22.7 combination ( $P$ values $<0.02$ and $<0.01$, respectively) (Fig. 2 a). This is consistent with the in vivo levels of rituximab in patients needed to achieve cytotoxic effects. To determine if this increase in cytotoxicity was due to apoptosis, Raji and Ramos NHL cells were treated as described earlier and apoptosis was assessed using a polycaspases FLICA kit. The percentage of apoptotic cells was significantly greater $(P<0.01)$ for Bs20x22 treatment in both Raji and Ramos cells when compared with combination rituximab/HB22.7 treatment (Fig. 2b). Combination rituximab/HB22.7 treatment did not significantly increase apoptosis over rituximab or HB22.7 treatment alone (Fig. 2b) and suggests that the Bs20x22 have apoptotic effects that are more potent than either $\mathrm{mAb}$ alone.

We next began to examine the mechanism for the enhanced in vitro cytotoxicity observed with Bs20x22 treatment. Both HB22.7 and rituximab activate the p38 signaling pathway, and it has been hypothesized that $\mathrm{p} 38$ activation may mediate cytotoxic effects. Therefore, the ability of Bs20x22 to activate various signaling proteins was compared with the parent mAbs. Western blot analysis demonstrated that Bs20x22 activates $\mathrm{p}-38$ to a much greater extent that either parent $\mathrm{mAb}$ alone (Fig. 3). Longer exposures revealed a relatively low level of $\mathrm{p} 38$ activation with either parent $\mathrm{mAb}$ alone, which may be explained by the fact that for the current study, the dose of each $\mathrm{mAb}$ was much lower than those used in previous studies. Examination of the ERK-1/2 and JNK signaling pathways revealed that there was no differential activation of these pathways with BS20x22.

We next sought to determine if the in vitro results translated into an improvement in efficacy in vivo. Xenograft bearing mice were treated with rituximab, HB22.7, combination rituximab/HB22.7, or Bs20x22 on days 14 , 21 , and 28 after tumor implantation. Mice treated with Bs20x22 had the smallest tumor volume of any group, although comparisons between groups were only significant for the Bs20x22 versus control and the Bs20x22 versus HB22.7 comparisons (Fig. 4a, Table 1). By the end of the study, Bs20x22-treated mice had tumors that were, on average, $50 \%$ smaller than tumors from combination rituximab/HB22.7-treated mice (Fig. 4a). In addition to smaller tumors, at the end of the study, Bs20x22-treated mice exhibited the greatest survival rate of any group (88\%) compared with combination rituximab/HB22.7 (75\%), rituximab (50\%), HB22.7 (25\%), and control (0\%) (Fig. 4b). This compares favorably to the survival seen in the same xenograft model treated with the hexavalent bispecific CD20/CD22 mAb [26]. Previous studies with HB22.7 and rituximab have shown more robust tumor volume reduction than that seen in this study [5, 16, 27, 29]. This can be explained by the fact that in order to maintain equivalent total $\mathrm{mAb}$ dosing between all groups, and in order to see any additive effects of rituximab/HB22.7 combination, less than optimal doses of rituximab and HB22.7 were used in this study. Previous pre-clinical studies with both rituximab and HB22.7 demonstrated superior efficacy when used to treat smaller tumors or when administered preemptively; therefore, we also compared Bs20x22 treatment in a preemptive model versus an established tumor model. Not surprisingly, mice treated with preemptive Bs20x22 had tumors that were, on average, $89 \%$ smaller than those in the established tumor Bs20x22 treatment group (Fig. 4c).

It is interesting to hypothesize how Bs20x22 exerts its effects. Studies using veltuzumab/epratuzumab-based CD20/CD22 BsAbs showed that these BsAbs increased antibody dependent cellular cytotoxicity (ADCC) but no complement-dependent cytotoxicity (CDC) when compared to parent mAbs $[25,26]$. In fact, all in vivo activity was abrogated by NK cell depletion. Cross-linking with these BsAbs causes translocation of $\mathrm{CD} 20, \mathrm{CD} 22$, and BCR into lipid rafts and the effects of BsAb-induced 
localization of BCR into lipid rafts resembles that caused by anti-IgM [25]. In addition, CD22 undergoes internalization once bound by ligand, while CD20 does not [33]. By cross-linking CD22 to CD20, a CD20/CD22 BsAb prevents CD22 internalization [25], which likely affects downstream signaling. Since our Bs20x22 uses rituximab and HB22.7, the mechanisms of Bs20x22's actions may differ from those of veltuzumab/epratuzumab-based BsAbs. However, it is clear that no matter what the platform, BsAbs exhibit novel mechanisms when compared to their parent mAbs. These mechanisms are an active area of exploration.

Our data suggest that $\mathrm{Bs} 20 \times 22$ is a more efficacious treatment for NHL than is combination rituximab/HB22.7 and possibly better than the veltuzumab/epratuzumab-based BsAbs. It is also important to note that Bs20x22 does not utilize an intact antibody/Fc and thus is likely not dependent on host immune effector mechanisms. This may be important in many lymphoma patients that are immunocompromised. In addition, the use of a BsAb eliminates the need for sequential administration of two separate mAbs, saving the patient's time. The NCI has recently approved and funded the humanization of HB22.7 through the Rapid Access Intervention Drug (RAID) Program. Given the pre-clinical potential of Bs20x22, this humanized HB22.7 can be tested in a new, humanized version of Bs20x22.

Acknowledgments This work was supported by the Schwedler Family Foundation and the deLeuze Non-toxic Cure for Lymphoma Fund.

Open Access This article is distributed under the terms of the Creative Commons Attribution Noncommercial License which permits any noncommercial use, distribution, and reproduction in any medium, provided the original author(s) and source are credited.

\section{References}

1. Ries L, Melbert D, Krapcho M, Stinchcomb D, Howlader N, Horner M, Mariotto A, Miller B, Feuer E, Altekruse S, Lewis D, Clegg L, Eisner M, Reichman M, Edwards BE (2008) SEER cancer statistics review, 1975-2005. National Cancer Institute, Bethesda, MD

2. Lindley C (1991) The lymphomas: Hodgkin's disease and nonHodgkin's lymphomas. Am Pharm NS 31:46-51

3. Schumer ST, Joyce RM (2003) Radioimmunotherapy for NonHodgkin's Lymphoma. Progress Oncol 46-72

4. Collins-Burow B, Santos ES (2007) Rituximab and its role as maintenance therapy in non-Hodgkin lymphoma. Expert Rev Anticancer Ther 7:257-273

5. Molina A (2008) A decade of rituximab: improving survival outcomes in non-Hodgkin's lymphoma. Annu Rev Med 59:237-250

6. Tedder TF, Tuscano J, Sato S, Kehrl JH (1997) CD22, a B lymphocyte-specific adhesion molecule that regulates antigen receptor signaling. Annu Rev Immunol 15:481-504

7. Sato S, Tuscano JM, Inaoki M, Tedder TF (1998) CD22 negatively and positively regulates signal transduction through the $\mathrm{B}$ lymphocyte antigen receptor. Semin Immunol 10:287-297
8. Tuscano JM, Riva A, Toscano SN, Tedder TF, Kehrl JH (1999) CD22 cross-linking generates B-cell antigen receptor-independent signals that activate the JNK/SAPK signaling cascade. Blood 94:1382-1392

9. Williamson JM, Grigor I, Smith ME, Holgate CS, O'Brien CJ, Morgan DR, Quirke P, Alison DL, Child JA, Bird CC (1987) Ploidy, proliferative activity, cluster differentiation antigen expression and clinical remission in high-grade non-Hodgkin's lymphoma. Histopathology 11:1043-1054

10. Wain SL, Braylan RC, Borowitz MJ (1987) Correlation of monoclonal antibody phenotyping and cellular DNA content in non-Hodgkin's lymphoma. The Southeastern Cancer Study Group experience. Cancer 60:2403-2411

11. Press OW, Leonard JP, Coiffier B, Levy R, Timmerman J (2001) Immunotherapy of Non-Hodgkin's lymphomas. Hematol Am Soc Hematol Educ Program 221-240

12. Engel P, Nojima Y, Rothstein D, Zhou LJ, Wilson GL, Kehrl JH, Tedder TF (1993) The same epitope on CD22 of B lymphocytes mediates the adhesion of erythrocytes, $\mathrm{T}$ and $\mathrm{B}$ lymphocytes, neutrophils, and monocytes. J Immunol 150:4719-4732

13. Engel P, Wagner N, Miller AS, Tedder TF (1995) Identification of the ligand-binding domains of CD22, a member of the immunoglobulin superfamily that uniquely binds a sialic aciddependent ligand. J Exp Med 181:1581-1586

14. Law CL, Aruffo A, Chandran KA, Doty RT, Clark EA (1995) Ig domains 1 and 2 of murine CD22 constitute the ligand-binding domain and bind multiple sialylated ligands expressed on $\mathrm{B}$ and $\mathrm{T}$ cells. J Immunol 155:3368-3376

15. Tuscano J, Engel P, Tedder TF, Kehrl JH (1996) Engagement of the adhesion receptor $\mathrm{CD} 22$ triggers a potent stimulatory signal for B cells and blocking CD22/CD22L interactions impairs T-cell proliferation. Blood 87:4723-4730

16. Tuscano JM, O’Donnell RT, Miers LA, Kroger LA, Kukis DL, Lamborn KR, Tedder TF, DeNardo GL (2003) Anti-CD22 ligand-blocking antibody HB22.7 has independent lymphomacidal properties and augments the efficacy of 90Y-DOTA-peptide-Lym-1 in lymphoma xenografts. Blood 101:3641-3647

17. Leonard JP, Goldenberg DM (2007) Preclinical and clinical evaluation of epratuzumab (anti-CD22 IgG) in B-cell malignancies. Oncogene 26:3704-3713

18. Leonard JP, Coleman M, Ketas J, Ashe M, Fiore JM, Furman RR, Niesvizky R, Shore T, Chadburn A, Horne H, Kovacs J, Ding CL, Wegener WA, Horak ID, Goldenberg DM (2005) Combination antibody therapy with epratuzumab and rituximab in relapsed or refractory non-Hodgkin's lymphoma. J Clin Oncol 23:5044-5051

19. Leonard JP, Schuster SJ, Emmanouilides C, Couture F, Teoh N, Wegener WA, Coleman M, Goldenberg DM (2008) Durable complete responses from therapy with combined epratuzumab and rituximab: final results from an international multicenter, phase 2 study in recurrent, indolent, non-Hodgkin lymphoma. Cancer 113:2714-2723

20. Strauss SJ, Morschhauser F, Rech J, Repp R, Solal-Celigny P, Zinzani PL, Engert A, Coiffier B, Hoelzer DF, Wegener WA, Teoh NK, Goldenberg DM, Lister TA (2006) Multicenter phase II trial of immunotherapy with the humanized anti-CD22 antibody, epratuzumab, in combination with rituximab, in refractory or recurrent non-Hodgkin's lymphoma. J Clin Oncol 24:3880-3886

21. Arndt MA, Krauss J, Kipriyanov SM, Pfreundschuh M, Little M (1999) A bispecific diabody that mediates natural killer cell cytotoxicity against xenotransplantated human Hodgkin's tumors. Blood 94:2562-2568

22. Holliger P, Brissinck J, Williams RL, Thielemans K, Winter G (1996) Specific killing of lymphoma cells by cytotoxic T-cells mediated by a bispecific diabody. Protein Eng 9:299-305

23. Repp R, van Ojik HH, Valerius T, Groenewegen G, Wieland G, Oetzel C, Stockmeyer B, Becker W, Eisenhut M, Steininger H, 
Deo YM, Blijham GH, Kalden JR, van de Winkel JG, Gramatzki M (2003) Phase I clinical trial of the bispecific antibody MDX$\mathrm{H} 210$ (anti-FcgammaRI $\mathrm{x}$ anti-HER-2/neu) in combination with Filgrastim (G-CSF) for treatment of advanced breast cancer. Br J Cancer 89:2234-2243

24. Loffler A, Kufer P, Lutterbuse R, Zettl F, Daniel PT, Schwenkenbecher JM, Riethmuller G, Dorken B, Bargou RC (2000) A recombinant bispecific single-chain antibody, CD19 x CD3, induces rapid and high lymphoma-directed cytotoxicity by unstimulated T lymphocytes. Blood 95:2098-2103

25. Qu Z, Goldenberg DM, Cardillo TM, Shi V, Hansen HJ, Chang CH (2008) Bispecific anti-CD20/22 antibodies inhibit B-cell lymphoma proliferation by a unique mechanism of action. Blood 111:2211-2219

26. Rossi EA, Goldenberg DM, Cardillo TM, Stein R, Chang CH (2009) Hexavalent bispecific antibodies represent a new class of anticancer therapeutics: 1. Properties of anti-CD20/CD22 antibodies in lymphoma. Blood 113:6161-6171

27. O'Donnell RT, Ma Y, McKnight HC, Pearson D, Tuscano JM (2009) Dose, timing, schedule, and the choice of targeted epitope alter the efficacy of anti-CD22 immunotherapy in mice bearing human lymphoma xenografts. Cancer Immunol Immunother 58:2051-2058

28. Carnahan J, Wang P, Kendall R, Chen C, Hu S, Boone T, Juan T, Talvenheimo J, Montestruque S, Sun J, Elliott G, Thomas J,
Ferbas J, Kern B, Briddell R, Leonard JP, Cesano A (2003) Epratuzumab, a humanized monoclonal antibody targeting CD22: characterization of in vitro properties. Clin Cancer Res 9:3982S$3990 \mathrm{~S}$

29. O'Donnell RT, Pearson D, McKnight HC, Ma YP, Tuscano JM (2009) Treatment of non-Hodgkin's lymphoma xenografts with the HB22.7 anti-CD22 monoclonal antibody and phosphatase inhibitors improves efficacy. Cancer Immunol Immunother 58:1715-1722

30. Poe JC, Fujimoto Y, Hasegawa M, Haas KM, Miller AS, Sanford IG, Bock CB, Fujimoto M, Tedder TF (2004) CD22 regulates B lymphocyte function in vivo through both ligand-dependent and ligand-independent mechanisms. Nat Immunol 5:1078-1087

31. Tuscano JM, Engel P, Tedder TF, Agarwal A, Kehrl JH (1996) Involvement of p72syk kinase, p53/56lyn kinase and phosphatidyl inositol-3 kinase in signal transduction via the human B lymphocyte antigen CD22. Eur J Immunol 26:1246-1252

32. Stein R, Belisle E, Hansen HJ, Goldenberg DM (1993) Epitope specificity of the anti-(B cell lymphoma) monoclonal antibody, LL2. Cancer Immunol Immunother 37:293-298

33. Haas KM, Sen S, Sanford IG, Miller AS, Poe JC, Tedder TF (2006) CD22 ligand binding regulates normal and malignant B lymphocyte survival in vivo. J Immunol 177:3063-3073 